REGARDS

SUR LECONOMIE ALLEMANDE

BULLETIN ECONOMIQUE DU CRAC

\section{Regards sur l'économie allemande}

Bulletin économique du CIRAC

$114 \mid 2014$

Varia

\title{
Transition énergétique en RFA
}

L'impact sur les réseaux électriques en Allemagne et en Europe

\section{Ulrike Hansen}

Traducteur : Isabelle Bourgeois

\section{OpenEdition}

\section{Journals}

Édition électronique

URL : http://journals.openedition.org/rea/4726

DOI : $10.4000 /$ rea.4726

ISSN : 1965-0787

Éditeur

CIRAC

Édition imprimée

Date de publication : 1 octobre 2014

Pagination : 29-39

ISSN : 1156-8992

Référence électronique

Ulrike Hansen, "Transition énergétique en RFA », Regards sur l'économie allemande [En ligne],

114 | octobre 2014, mis en ligne le 01 octobre 2016, consulté le 01 mai 2019. URL : http://

journals.openedition.org/rea/4726 ; DOI : 10.4000/rea.4726 


\section{Transition énergétique en RFA}

\section{- l'impact sur les réseaux électriques en Allemagne et en Europe -}

\section{Ulrike Hansen}

La réorientation de la transition énergétique allemande (sur l'impératif de réformes, voir REA 112/2014) est en cours depuis le début de 2014. Avec l'entrée en vigueur, le 1er août 2014, de la loi de révision de la Loi de promotion des énergies renouvelables (Erneuerbare Energien-Gesetz, EEG), les mesures suivantes ont été mises en œuvre : définition d'objectifs de production d'énergie de source renouvelable, révision des critères de soutien aux énergies renouvelables et obligation de vente directe sur le marché de l'électricité. Par ailleurs, le ministère fédéral de l'Economie et de l'Energie a publié le 24-06-2014 un "Agenda énergie en 10 points » (10 Punkte-Energie-Agenda) qui vise la coordination des approches, et des calendriers afférents, à savoir : réforme de la Loi EEG, mise en œuvre conjointe du Paquet Climat-Energie 2030 au sein de l'UE, révision du système européen d'échange de quotas d'émission de $\mathrm{CO}_{2}$, nouvelle approche de l'organisation du marché de l'électricité et de coordination des mix énergétiques, définition d'une stratégie d'efficacité énergétique dans le $B T P$, développement du réseau allemand de transport et de distribution d'électricité, suivi annuel de l'avancée de la transition énergétique (monitoring) et coopération renforcée des plateformes de recherche en la matière.

L'accélération du développement du réseau électrique est un élément central de la transition énergétique allemande depuis son lancement voici bientôt quinze ans. Après l'arrêt de huit réacteurs (7+1) intervenu en 2011 à la suite du moratoire de sortie accélérée du nucléaire, le volume d'électricité d'origine nucléaire injecté dans le réseau a baissé de quelque $9700 \mathrm{MW}$, ce qui a profondément modifié la structure du flux de charge, se traduisant par une hausse des importations d'électricité (cette situation s'est toutefois inversée entretemps, l'Allemagne étant aujourd'hui exportatrice) et des flux acheminés d'est en ouest. Cet impact du moratoire sur l'abandon du nucléaire d'ici 2022 à la fois sur les réseaux de transport et la sécurité de l'approvisionnement vient rappeler l'impératif du développement des réseaux.

Cet impératif est paneuropéen. Mais la coopération au sein de l'UE entre les exploitants de réseaux est-elle satisfaisante? Le Paquet infrastructure adopté dans l'UE en 2010 tient-il suffisamment compte de cette priorité ? Depuis toujours, la sécurité durable de l'approvisionnement requiert l'adaptation permanente des réseaux. La transition énergétique - en Allemagne, en France - est venue rappeler combien cette question est cruciale.

Depuis plus d'une décennie, la question de l'extension des réseaux joue un rôle central dans la politique de transition énergétique allemande. Avant l'institution d'une régulation propre au marché de l'électricité en 2005, le calcul prévisionnel des besoins de production, de la consommation, et des impératifs de développement des réseaux s'effectuait au sein même de chaque entreprise du secteur de l'électricité ; ces électriciens étaient alors des sociétés verticalement intégrées. Or la libéralisation (1999) a eu pour effet la séparation des activités de production, de transport et de distribution (unbundling), de même que la multiplication des acteurs sur le marché de la production d'électricité. A cela est ve-

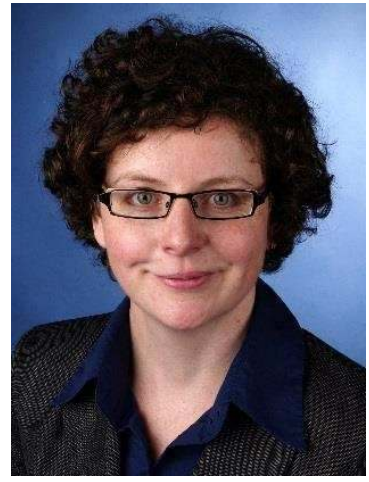

Ulrike Hansen, chargée de mission Relations internationales en énergie, Bundesnetzagentur.

L'auteur s'exprime ici en son nom personnel.
Une prise de conscience précoce 
nu s'ajouter le choix d'un mix énergétique accordant une place croissante à l'électricité produite à partir de sources renouvelables. II est alors apparu dès 2003 que cette évolution rendait incontournable un effort accru de développement du réseau à très haute tension et que celui-ci devait être accompagné par les pouvoirs publics. Comme l'actuelle Autorité de régulation : la Bundesnetzagentur (BNetzA), n'avait alors pas encore de compétences énergétiques, c'est l'Agence allemande de l'énergie (Deutsche Energieagentur, dena) qui fut chargée par le ministère fédéral de l'Environnement de rédiger un rapport sur la planification des réseaux à l'horizon 2020, intégrant l'éolien onshore et offshore (rapport « Energiewirtschaftliche Planung für die Netzintegration von Windenergie in Deutschland an Land und Offshore bis zum Jahr 2020 », 2005).

\section{Le marché de l'électricité allemand - données clés}

En Allemagne, on compte 4 exploitants de réseaux de transport d'électricité : Amprion, Tennet, Transnet BW et 50 Hertz. Ces sociétés sont toutes de droit privé, au statut de sarl $(\mathrm{GmbH})$. Jusqu'ici, Amprion, Transnet BW et 50 Hertz ont obtenu la certification délivrée par l'autorité de régulation, la BNetzA, de même que l'opérateur de réseaux offshore Tennet Offshore mbH.

Le réseau de répartition d'électricité est géré actuellement par 889 sociétés.

La gestion des réseaux est totalement séparée des activités que sont la production d'électricité et sa distribution au consommateur final. La part de marché des 4 plus grandes sociétés de production d'électricité s'élève à environ $76 \%$, soit 81,4 GW (état au 31-12-2012). Alors que la production d'électricité de source conventionnelle est tombée à 437,7 TWh en 2012 (soit -8,1 TWh par rapport à 2011), celle issue de sources renouvelables a progressé de 19,1 TWh pour s'établir à 138,9 TWh. L'Allemagne est exportateur net d'électricité et occupe, en sa qualité de pays de transit, le rôle de plaque tournante dans le système central de couplage des marchés européens (Bundesnetzagentur, Monitoringbericht, 2013).

2005 : obligation légale pour les gestionnaires de réseaux de les développer...

... mais trop de lourdeurs administratives

2006/11: simplification des procédures en trois temps
La loi sur l'approvisionnement en gaz et en électricité adoptée en 2005 et surnommée "Loi sur l'économie de l'énergie " (Energiewirtschaftsgesetz, EnWG) fait obligation aux opérateurs de réseau " d'entretenir et d'optimiser en fonction des besoins, de renforcer et de développer le réseau dans la mesure de ce qui est économiquement raisonnable » (§ 11, al. 1 loi EnWG). Ils peuvent pour cela déposer auprès de la Bundesnetzagentur (BnetzA), des demandes de soutien à l'investissement pour des projets individuels de développement du réseau. La loi EnWG confie également aux exploitants de réseaux de transport la mission explicite de planifier le réseau de transport. Ceux-ci devaient déposer tous les deux ans devant la BnetzA un rapport sur l'état de leur planification. Depuis 2011, ce rapport doit être remis tous les ans.

Or l'extension et la modernisation du réseau a pris beaucoup de retard, du fait entre autres de la lenteur des procédures administratives d'autorisation. Face à la persistance des lacunes et pénuries constatées, le législateur a donc dû prendre une série de mesures pour accélérer le développement du réseau.

Une première Loi visant à accélérer les procédures de planification d'infrastructures a ainsi été adoptée le 09-12-2006. Entrée en vigueur le 17-12-2006, elle modifie les procédures administratives et réglementaires d'autorisation de certaines infrastructures de transport de gaz et d'électricité contenues dans la loi EnWG, importantes notamment pour sécuriser l'approvisionnement. Elle facilite les procédures réglementaires d'autorisation.

Une deuxième Loi accélérant le développement des réseaux (Netzausbaubeschleunigungsgesetz, NABEG) a été adoptée le 21-08-2009, parallèlement à la Loi sur l'extension des lignes de transport d'énergie (Energieleitungsausbaugesetz, EnLAG). Une telle loi était devenue nécessaire parce que la précédente n'avait pas suffi à réduire les retards pris tant au niveau de la planification que de l'extension réalisée du réseau. Le pivot de la modification est donc l'inscription des différents projets - notamment des projets d'intérêt commun européen - au sein d'une loi de programmation prévisionnelle. Auparavant, le contrôle du bien-fondé de ces projets d'extension s'effectuait dans le cadre de plusieurs procédures administratives relevant de la compétence des Länder : la procédure de l'aménagement et la procédure réglementaire d'autorisation permettant la construction des lignes, et sur l'aspect du financement, la procédure d'octroi des budgets d'investissement par la Bundesnetzagentur. 
La dernière révision de la Loi NABEG a été adoptée en 2011. Elle simplifie en profondeur les processus d'harmonisation des scenarii, de planification des réseaux et les procédures d'autorisation (procédure d'approbation des grands projets publics) tout en en réduisant nettement la durée.

Depuis 2011, la planification des réseaux de transport d'électricité s'effectue de la manière suivante ( $\S 12$ al. a-e EnWG) : les exploitants de réseaux allemands présentent chaque année un schéma-cadre conjoint. II constitue la base sur laquelle s'effectue la planification de l'ensemble des réseaux, qu'ils soient onshore ou offshore. II comprend au moins trois projections (scenarii) censés correspondre aux évolutions probables des objectifs de politique énergétique fixés par le gouvernement fédéral au cours des dix années à venir. L'un des scenarii porte sur les deux décennies à venir. Les exploitants de réseaux posent donc, pour chacun des scenarii, des hypothèses de travail en ce qui concerne la production, la distribution et la consommation d'électricité, de même que les échanges avec les Etats voisins, et ils prennent en considération également les programmes d'investissement concernant l'infrastructure des réseaux européens. Ce projet de schéma-cadre est remis à la Bundesnetzagentur qui le soumet à une procédure de consultation publique à l'issue de laquelle, et en tenant compte des avis émis lors de cette dernière, l'Autorité de régulation approuve annuellement ce schéma-cadre.
2011, Loi NABEG : optimisation de la planification des réseaux

Objectifs du schéma-cadre approuvé par la Bundesnetzagentur le 30-08-2013 et relatif au Plan de développement des réseaux 2014 ainsi qu'au Plan de développement des réseaux offshore 2014

\begin{tabular}{|lrrrrr|}
\hline & Référence & Scénario A & Scénario B & Scénario B & Scénario C \\
Capacité de production installée [GW] & $\mathbf{2 0 1 2}$ & $\mathbf{2 0 2 4}$ & $\mathbf{2 0 2 4}$ & $\mathbf{2 0 3 4}$ & $\mathbf{2 0 2 4}$ \\
Nucléaire & & & & & \\
Lignite & 12,1 & 0,0 & 0,0 & 0,0 & 0,0 \\
Houille & 21,2 & 16,0 & 15,4 & 11,3 & 15,4 \\
Gaz naturel & 25,4 & 27,2 & 25,8 & 18,4 & 25,8 \\
Pétrole & 27,0 & 23,3 & 28,2 & 37,5 & 28,2 \\
Centrales de pompage & 4,0 & 1,8 & 1,8 & 1,1 & 1,8 \\
Autres sources conventionnelles & 6,4 & 10,0 & 10,0 & 10,7 & 10,0 \\
Total prod. de source conventionnelle & 4,1 & 3,7 & 3,7 & 2,7 & 3,7 \\
Eolien onshore & $\mathbf{1 0 0 , 2}$ & $\mathbf{8 2 , 0}$ & $\mathbf{8 4 , 9}$ & $\mathbf{8 1 , 7}$ & $\mathbf{8 4 , 9}$ \\
Eolien offshore & 31,0 & 49,0 & 55,0 & 72,0 & 87,4 \\
Photovoltaïque & 0,3 & 11,5 & 12,7 & 25,3 & 16,1 \\
Biomasse & 33,1 & 54,8 & 56,0 & 59,5 & 58,6 \\
Hydraulique & 5,7 & 8,3 & 8,7 & 9,2 & 7,8 \\
Autres sources renouvelables & 4,4 & 4,5 & 4,7 & 5,0 & 4,2 \\
Total prod. de source renouvelable & 0,8 & 0,9 & 1,5 & 2,3 & 1,3 \\
Total production & $\mathbf{7 5 , 3}$ & $\mathbf{1 2 9 , 0}$ & $\mathbf{1 3 8 , 6}$ & $\mathbf{1 7 3 , 3}$ & $\mathbf{1 7 5 , 4}$ \\
Consommation nette prévisible [TWh] *) & $\mathbf{1 7 5 , 5}$ & $\mathbf{2 1 1 , 0}$ & $\mathbf{2 2 3 , 5}$ & $\mathbf{2 5 5 , 0}$ & $\mathbf{2 6 0 , 3}$ \\
Pic annuel [GW] *) & $\mathbf{5 4 0 , 3}$ & $\mathbf{5 3 5 , 4}$ & $\mathbf{5 3 5 , 4}$ & $\mathbf{5 3 5 , 4}$ & $\mathbf{5 3 5 , 4}$ \\
\hline
\end{tabular}

Source : www.netzausbau.de/cln_1412/DE/Bedarfsermittlung/Charlie/SzenariorahmenCharlie/SzenariorahmenCharlie-node.html. *) Y inclus pertes en ligne dans le réseau de distribution.

Sur la base du schéma-cadre ainsi retenu, les exploitants de réseau se mettent d'accord, chaque année, sur des projets de développement des réseaux (NetzVaste entwicklungspläne, NEP), onshore et offshore (§12b EnWG). Ces plans annuels doivent contenir toutes les mesures opportunes visant l'optimisation des réseaux selon les besoins, l'accroissement de leur capacité et leur extension. Celles-ci sont approuvées dès lors qu'elles sont nécessaires pour assurer le fonctionnement sûr et fiable des réseaux au cours des dix années à venir. Ces projets annuels sont eux aussi soumis à consultation publique avant d'être transmis pour approbation à l'Autorité de régulation BNetzA.

Après examen, cette dernière les soumet à son tour à consultation. Afin de prendre en considération dès l'amont l'impact environnemental de ces projets annuels, la BNetzA effectue un contrôle stratégique de conformité avec la 
Programmation bottom-up des besoins

protection de l'environnement du Plan fédéral de programmation des besoins (Bundesbedarfsplan). Sont ainsi évaluées, pour chacun des projets, les répercussions sur la santé humaine, la biodiversité et l'environnement que pourrait avoir la construction de lignes électriques, qu'elles soient aériennes ou souterraines, qu'elles transportent du courant continu ou triphasé. Les conclusions de ces travaux sont ensuite résumées dans un Rapport sur l'environnement soumis lui aussi à consultation publique. La BNetzA prend ensuite en considération les avis prononcés par les citoyens et les administrations lorsqu'elle entérine les Projets de développement des réseaux onshore et offshore (NEP). Ceux-ci, de même que le Rapport sur l'environnement, constituent les deux piliers sur la base desquels s'effectue la programmation annuelle du développement des réseaux.

En outre, si des modifications conséquentes sont intervenues, mais au minimum tous les trois ans, l'Autorité de régulation soumet au gouvernement fédéral les NEP qu'elle a entérinés et son Rapport environnemental pour alimenter le projet de Plan fédéral de programmation des besoins que le gouvernement fédéral soumet au vote du Parlement tous les trois ans au minimum également. C'est ainsi que prend forme la Loi de programmation fédérale des besoins (Bundesbedarfsplangesetz).

Lignes projetées dans le cadre de la Loi de programmation fédérale des besoins 2013

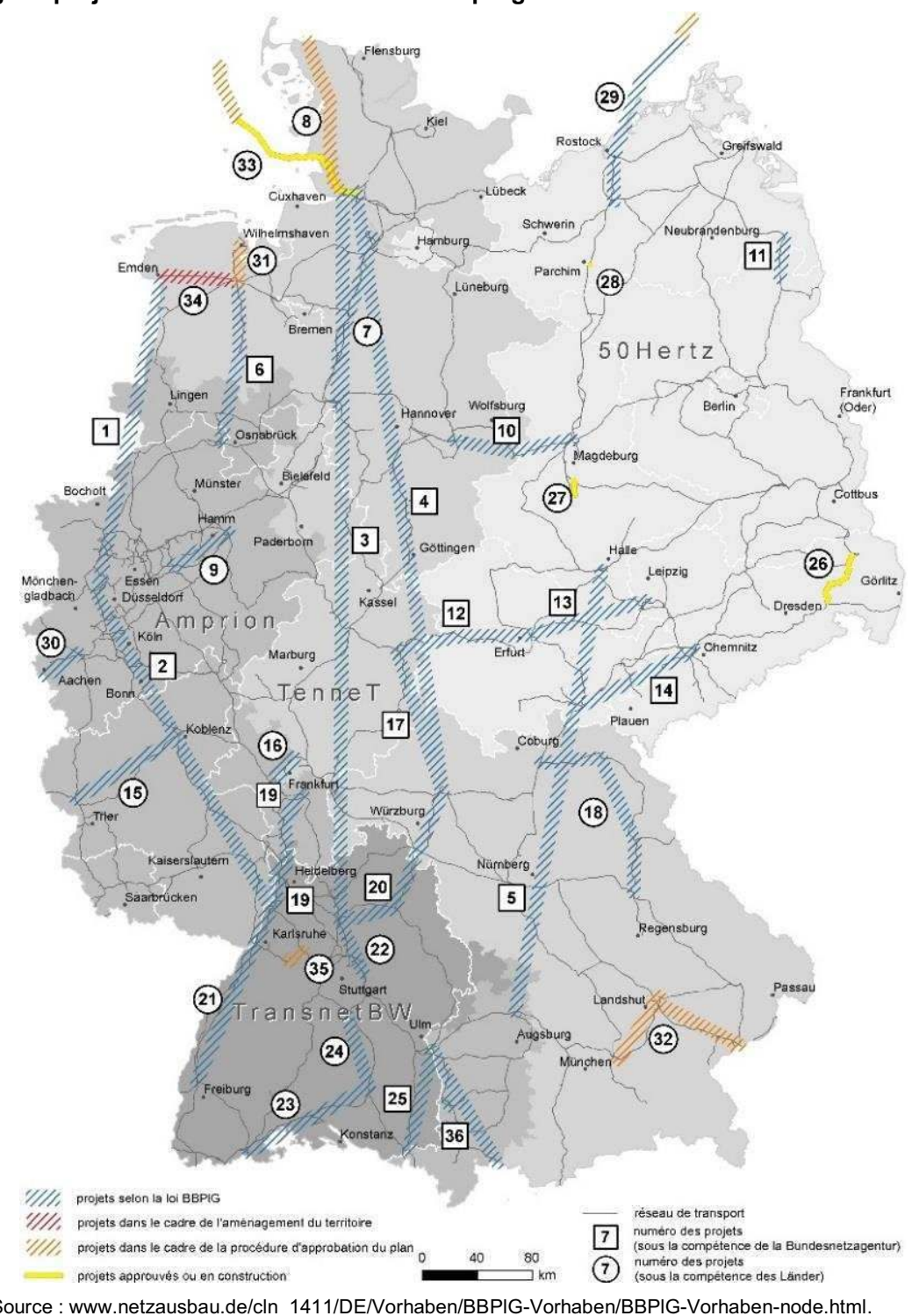


La Loi de programmation fédérale des besoins adoptée le 23-07-2013 a pour la première fois défini, sous l'angle des impératifs de politique énergétique, la nécessité du développement des réseaux et les besoins afférents tels que prévus par la loi, les rendant de la sorte contraignants pour les exploitants de réseaux, y compris dans le cadre de la procédure réglementaire d'autorisation (§§ 4343d EnWG et §§ 18-24 NABEG).

Ces dispositions suppriment tout risque d'incertitude lié à l'appréciation de l'Autorité de régulation, qu'il s'agisse de l'agrément des coûts programmés pour les travaux d'extension du réseau ou de l'octroi des autorisations afférentes. Non contentes de cela, elles font obligation aux exploitants de réseaux de transport de réaliser effectivement les projets d'extension et d'aménagement contenus dans les Projets de développement approuvés par la BNetzA. La Loi de programmation des besoins 2013 apporte ainsi aux exploitants de réseaux une sécurité juridique maximale en ce qui concerne les investissements requis.

Dans un deuxième temps, les exploitants de réseaux de transport peuvent faire auprès de l'Autorité de régulation une demande d'attribution de corridors à l'intérieur des tracés programmés par la Loi fédérale de programmation des besoins. Cette procédure, dite de planification technique fédérale (Bundesfachplanung), s'applique aux liaisons à très haute tension traversant les frontières des Länder ou des Etats limitrophes. Ces corridors constituent alors la base de la procédure réglementaire d'autorisation pour la construction des lignes, prévue par la procédure de planification des réseaux confiée notamment à la BNetzA (§§ 4 sq. NABEG). Ces deux procédures - planification technique fédérale et procédure réglementaire d'autorisation - incluent elles aussi une large consultation du public.

Une consultation d'une telle ampleur a pour objectif foncier d'engager le dialogue avec toutes les parties intéressées et tous les citoyens concernés et ce, le plus tôt possible afin de pouvoir rendre intelligibles les projets d'infrastructures, de permettre à l'opinion d'exprimer ses craintes et d'en débattre. C'est ainsi que, depuis 2011, l'Autorité de régulation organise, dans l'ensemble de l'Allemagne, nombre de manifestations destinées à informer les citoyens sur les schémas-cadre, les projets de développement des réseaux et les rapports environnementaux, ainsi que des débats sur les technologies, la protection de l'environnement ou l'état de la recherche.

\section{Impact sur les réseaux : la question de la sécurité de l'approvisionnement}

Outre la planification de l'extension des réseaux et la simplification des procédures réglementaires d'autorisation, une autre question encore est centrale pour le bon déroulement de la transition énergétique : celle de la sécurité de l'approvisionnement. L'abandon progressif de la production d'électricité de source nucléaire exige en effet que soit préservée une capacité de production suffisante pour couvrir les besoins en électricité. II implique également que soit garantie la stabilité du réseau lorsque, du fait de la volatilité de la production des énergies renouvelables, survient un déséquilibre entre production injectée et énergie soutirée. Et que, enfin, les réseaux soient sécurisés contre l'intervention de tiers. Pour répondre à ces exigences, les exploitants de réseaux de transport et l'Autorité de régulation veillent à garantir la capacité de production de source conventionnelle nécessaire pour les années à venir.

Cette mission s'effectue d'une part via un suivi de l'arrêt des diverses centrales. A cet effet, les exploitants de sites de production et de stockage sont tenus de notifier aux exploitants de réseaux de transport concernés et à la BNetzA leur projet de fermeture au moins 12 mois avant l'arrêt de leurs installations. Celle-ci n'intervient pas nécessairement à la date théoriquement prévue. Car si l'exploitant du réseau de transport concerné justifie de l'importance pour le système du
Sécurité juridique de la Loi fédérale de programmation des besoins

Lignes à très haute tension : attribution de corridors ...

... et consultation du public avant mise en chantier

Suivi minutieux de l'arrêt des centrales 
Evaluation permanente des besoins en capacités de réserve

Lecture européenne des capacités de production

ENTSO-E : planification du développement du réseau européen site à fermer et que l'Autorité de régulation l'agrée ( $\$ 13$ a al. 2 EnWG), la fermeture est ajournée. Une centrale ou un site sont jugés importants pour le système lorsque l'arrêt définitif de la centrale ou du site en question entraînerait une mise en danger ou une perturbation non négligeables de la sécurité ou de la fiabilité du système d'approvisionnement en électricité, et qu'il ne pourrait y être remédié d'aucune manière. Si la BNetzA donne son agrément à la catégorisation ainsi effectuée par l'exploitant du réseau de transport, la fermeture du site en question est repoussée, de 24 mois au maximum.

D'autre part, dans l'objectif d'assurer la stabilité du réseau, les exploitants de réseaux de transport doivent évaluer les besoins prévisionnels en matière de centrales de réserve et faire valider leurs prévisions par la BNetzA. La capacité de production ainsi mise en réserve est destinée à pallier une surcharge éventuelle du réseau. II s'agit là d'une mesure transitoire destinée à assurer la sécurité de l'approvisionnement tant que le réseau électrique, encore insuffisamment développé, est fragilisé par l'injection accrue d'électricité de source renouvelable (volatilité) et l'arrêt progressif de centrales conventionnelles. Les centrales de réserve représentent ainsi une assurance supplémentaire pour la stabilisation du réseau dans le cas où est épuisé le potentiel de mesures de redispatching de l'ensemble des centrales existant sur le marché. Ces mesures ont pour but d'éviter une surcharge possible d'une partie du réseau : si une congestion est prévisible dans un segment précis du réseau, les centrales en amont de ce segment sont tenues d'injecter moins d'électricité dans le réseau et celles situées en aval d'augmenter la puissance injectée. De cette manière (redispatching) est créé un flux de charge qui élimine la congestion.

Les dernières prévisions des exploitants de réseau, validées le 02-05-2014 par la BNetzA, sont publiées en ligne : "Pour l'hiver 2014/15, la puissance de production nécessaire à fournir par les centrales de réserve s'élève à $3091 \mathrm{MW}$. Pour la période 2015/16, elle est de 6000 MW, puis de 7000 MW pour la période 2017/18. " Une part importante de cette puissance de production est d'ores et déjà garantie par le biais d'engagements contractuels ou d'obligations légales: $3027 \mathrm{MW}$ pour l'hiver 2014/15 et respectivement $4561 \mathrm{MW}$ et $3924 \mathrm{MW}$ pour les années suivantes. Au vu du potentiel du marché des centrales de production en Allemagne comme dans les Etats limitrophes dans I'UE, la puissance manquante devrait être couverte sans difficulté.

Cela permet de mieux prendre mesure de l'ampleur de l'impact de la transition énergétique allemande et de comprendre le rôle crucial que joue dans ce contexte le développement des réseaux électriques. Quant au fait qu'il soit possible de trouver hors des frontières allemandes des centrales de réserve et de conclure des contrats d'approvisionnement avec elles, il révèle la dimension européenne de la transition énergétique allemande. Nous nous contenterons ici d'éclairer cette dimension sous l'angle des réseaux.

\section{Le contexte européen...}

La progression des énergies renouvelables n'affecte pas seulement les réseaux en Allemagne, mais aussi dans les Etats limitrophes. Par nature, les flux électriques allant du nord au sud de l'Allemagne se répercutent en effet sur les réseaux en Pologne et en République tchèque à l'est, aux Pays Bas, en Belgique et en France à l'ouest. Ces flux de courant en boucle (loopflows) qui existent dans tous les pays posent un défi paneuropéen qui a gagné en importance avec la hausse de la part du courant volatile injecté dans les réseaux au fil de l'essor des renouvelables. La seule mesure permettant d'y répondre est le développement conséquent du réseau électrique européen.

La planification du développement du réseau européen est effectuée, pour une période de dix ans, par le Réseau européen des gestionnaires de réseaux de transport d'électricité : le European Network of Transmission System Operators 
for Electricity (ENTSO-E). Un tel schéma de développement décennal (TenYear-Network-Development-Plan : TYNDP) a été adopté pour la première fois en 2010, et la troisième édition est attendue pour la fin 2014. Or dès 2009, et sans la moindre contrainte légale, les gestionnaires de réseaux de l'UE, réunis au sein de l'Union pour la coordination du transport de l'électricité (UCTE), avaient adopté un programme de développement (le Transmission Development plan-Development of interconnections); mais à l'époque, il s'agissait moins d'une gestion concertée que d'un simple assemblage des plans de développement nationaux respectifs.

L'approche de la planification paneuropéenne des réseaux a considérablement progressé depuis 2009. La convergence des schémas de développement européens est désormais assurée grâce au Réseau européen ENTSO-E et contrôlée à la fois par les autorités de régulation nationales et par l'Agence pour la coopération des régulateurs de l'énergie (ACER). Le dernier schéma décennal (TYNDP 2012) adopté par l'ENTSO-E a permis également de sélectionner des "projets d'intérêt commun " (PIC). Ce choix s'effectue sur la base du Règlement TEN-E (Règlement (UE) n³47/2013 du 17-04-2013 concernant des orientations pour les infrastructures énergétiques transeuropéennes).

Ces PIC sont des projets de construction d'infrastructures électriques, gazières ou pétrolières transfrontière ou dont l'impact se fait sentir substantiellement dans au moins deux Etats membres. Les dispositions de ce Règlement visent à promouvoir et à accélérer l'interconnexion des réseaux au sein de l'UE. La sélection des projets d'intérêt commun s'effectue depuis février 2012 dans le cadre d'une vaste procédure qui a abouti à l'adoption, par la Commission européenne, d'une première liste conjointe de PIC et son inscription en annexe du Règlement délégué (UE) $n^{\circ} 1391 / 2013$ de la Commission (14-10-2013). Dans cette liste de 248 projets (« liste de l'UE »), 20 concernent des infrastructures électriques ( 5 gazières et 2 pétrolières) en lien direct avec l'Allemagne.

Une telle approche n'est certes pas nouvelle : le programme Réseaux transeuropéens d'énergie (RTE-E) avait vu le jour en 1995 ; ses dernières orientations décidées en 2006 (Décision $n^{\circ}$ 1364/2006/CE du 06-09-2006) définissaient déjà 42 « projets d'intérêt commun » $(\mathrm{PIC})$, c'est-à-dire portant sur des ouvrages de connexion internationale ou nationale, dès lors que ceux-ci ont des répercussions sur les capacités d'interconnexion transfrontalières. Le fait que le nombre de ces PIC a plus que quintuplé dans I'UE depuis 2006 suffit à révéler l'importance et, plus encore, l'urgence du développement du réseau européen de l'électricité.

Cet objectif figure sur l'agenda de la Commission depuis 1996 (voir Nies, 2009). Depuis la libéralisation des marchés de l'électricité en Europe, les échanges entre les différents marchés de gros et de détail nationaux ont considérablement augmenté sous l'effet notamment de l'amélioration des conditions réservées au commerce transfrontalier, de même que se sont multipliés les flux qui transitent par les lignes d'interconnexion transfrontalières (Bundesnetzagentur, 2008). Pour prendre en considération cette évolution, lors du Conseil européen de Barcelone des 15/16-03-2002, les chefs de gouvernement se sont fixé pour objectif une capacité de transport (en MW) des interconnexions d'au moins $10 \%$ de la capacité de production nationale installée. Si le choix d'un critère de $10 \%$ n'a pas été explicité, il n'en traduit pas moins un certain engagement dans la solidarité énergétique européenne et dans la libéralisation du secteur de l'électricité (Nies, 2009).

Cet objectif 2005, dont le financement relevait pour l'essentiel des entreprises concernées, n'a toujours pas été atteint dans l'ensemble de l'UE (Conseil de I'Union européenne, Bruxelles, 12-12-2013, 17755/13 ENER 584). En Allemagne, le taux d'interconnexion atteignait plus de $14,4 \%$ en 2004 et 13,3 \% en 2008 (Bundesnetzagentur, Monitoringbericht 2007 et Monitoringbericht 2009). Bien que l'Allemagne dépasse ainsi le seuil de $10 \%$ fixé, et indépendamment
Définition de " projets d'intérêt européen »

Accroître la capacité des interconnexions transfrontalières 
2014 : la Stratégie européenne pour la sécurité énergétique...

... se conjugue avec le paquet climat-énergie

Respect du principe de subsidiarité

Transition énergétique, une préoccupation partagée

Investissements vertigineux pour la décarbonisation

Nécessité d'une grande prévisibilité de cet engagement européen, l'Autorité de régulation avait clairement souligné, dans son premier rapport d'évaluation des rapports sur l'état des réseaux et leur développement remis par les exploitants de réseaux allemands publié en 2008, la nécessité de multiplier les interconnexions aux frontières.

Au début de 2014 , la crise en Ukraine a replacé au centre de la politique énergétique européenne la sécurité de l'approvisionnement et le développement du réseau d'infrastructures. La Commission a présenté le 28-05-2014 sa Stratégie européenne pour la sécurité énergétique qui préconise une série de mesures portant notamment sur la réduction de la dépendance des importations de gaz. En ce qui concerne l'électricité, elle propose à l'horizon 2030 un objectif de $15 \%$ de la capacité de production nationale installée (Communication de la Commission au Parlement européen et au Conseil, Bruxelles, 2805-2014, COM(2014) 330 final).

Cet objectif visant à renforcer la sécurité énergétique se conjugue avec ceux proposés à l'horizon 2030 par la Commission dans son paquet climat-énergie présenté le 22-01-2014 (http://ec.europa.eu/energy/doc/2030/com_2014_15_ en.pdf). II s'agit de deux objectifs contraignants : réduction de $40 \%$ des émissions de gaz à effet de serre et une part de $27 \%$ d'énergies renouvelables dans la consommation totale dans I'UE (cet objectif ne sera que volontaire à l'échelon des Etats membres). Ces propositions sont actuellement en cours de discussion et doivent être adoptés lors du Conseil européen d'octobre 2014. La Commission vient de proposer en outre le 23-07-2014 d'accroître de 30 \% d'ici 2030 l'efficacité énergétique. Lors du même Conseil européen, il sera décidé si ce taux est maintenu et s'il aura un caractère contraignant ou non (http://ec. europa.eu/transparency/regdoc/rep/1/2014/FR/1-2014-520-FR-F1-1. Pdf).

La Commission ne prévoit aucun objectif pour le développement des énergies renouvelables dans chacun des Etats membres, préférant préserver leur liberté en ce qui concerne la vitesse avec laquelle ils choisissent de développer la part des renouvelables dans la production d'électricité. Cette flexibilité s'impose d'autant plus que ce développement entraîne - effet non négligeable - la nécessité d'aménager les réseaux de transport d'électricité.

Du point de vue allemand, il est primordial que l'UE mène une politique globale et ambitieuse en matière de climat et d'énergie au-delà de l'horizon 2020. Du fait de la priorité qu'elle accorde à la montée en puissance des énergies renouvelables, l'Allemagne joue assurément un rôle de pionnier. Or la Commission, dans sa Feuille de route pour l'énergie à l'horizon 2050, présentée en mars 2011, prône une quasi-totale "décarbonisation du système énergétique " de l'UE : d'ici 2050, les émissions de gaz à effet de serre dans l'UE doivent être réduites de $80 \%$ par rapport à 1990 (http://ec.europa.eu/energy/energy2020/ roadmap/doc/com_2011_8852_fr.pdf). La transition énergétique s'est donc imposée comme une préoccupation partagée au sein de l'UE ; dans ce contexte, l'impact sur les réseaux électriques de la politique menée par l'Allemagne en la matière est un exemple qui montre son impact sur le réseau européen d'électricité.

Selon l'édition 2014 du World Energy Investment Outlook présenté par l'Agence internationale de l'Energie (IEA), les investissements requis en Europe d'ici 2035 pour remplacer les installations obsolètes et atteindre l'objectif de décarbonisation s'élèvent à 2200 milliards $\$$ US (au taux de $1 \$=0,78 €$ ). Le Réseau ENTSO-E pour sa part estime le montant des investissements nécessaires au développement des réseaux tel que prévu dans le projet TYNDP 2014 à 150 milliards $€$ d'ici 2030 . Ces estimations montrent bien l'ampleur des travaux d'infrastructure qu'impliquent le remplacement des sources d'énergie conventionnelles par des renouvelables, le raccordement aux réseaux de l'électricité ainsi produite, comme son transport vers les postes électriques.

L'élément clé en est la multiplication des interconnexions entre les réseaux des Etats membres de I'UE : ENTSO-E estime que la capacité d'interconnexion doit 
doubler d'ici 2030. Les chantiers prioritaires sont le raccordement de la péninsule ibérique, de l'Irlande et de la Grande-Bretagne au continent européen, de même que celui des Etats baltes (Lituanie, Estonie et Lettonie). Et l'ENTSO-E rappelle avec insistance l'importance revenant à la planification du développement des réseaux pour atteindre les objectifs climatiques et d'essor des énergies renouvelables d'ici 2030. Les principaux défis à relever dans ce contexte sont d'une part l'accélération comme la simplification des procédures réglementaires d'autorisation et, d'autre part, une large adhésion des citoyens à l'extension des réseaux de transport d'électricité. Mais ce qui importe avant tout, c'est l'existence de conditions-cadre stables pour le refinancement des projets d'infrastructures, rappelle l'ENTSO-E.

\section{... et les perspectives}

En France, le Rapport Bizet publié en 2014 par le Sénat souligne lui aussi la composante européenne de la transition énergétique en France et en Allemagne et plaide pour une coopération franco-allemande plus poussée dans le domaine des infrastructures dans l'objectif de l'interconnexion des deux réseaux nationaux. Ainsi, une programmation conjointe des investissements par les exploitants de réseaux respectifs, puis l'élargissement de cette coopération à ceux d'autres pays, permettrait de pallier les insuffisances actuelles.

A l'échelon européen, les gestionnaires de réseaux, pour leur part, ont considérablement intensifié ces dernières années leur coopération, institutionnalisée par le Réseau ENTSO-E qui réunit 41 d'entre eux, collecte les statistiques nationales, et dont une des missions est la planification conjointe du réseau électrique européen. La qualité de ses projets biennaux TYNDP s'est ainsi nettement améliorée depuis 2010. Mais des progrès restent à faire dans ce domaine de coopération; l'Agence pour la coopération des Autorités de régulation ACER, ainsi que les Autorités de régulation nationales, ont dressé une série de pistes en ce sens. Quoi qu'il en soit, rien actuellement n'incite à penser que les plans de développement des exploitants de réseaux dans l'UE sont insuffisamment coordonnés ou inefficients.

L'Allemagne est pionnière en Europe en ce qui concerne la transition énergétique, mais aussi l'adéquation de la qualité de sa programmation des réseaux avec les besoins, régulièrement évaluée par l'Autorité de régulation. Or les planifications européenne et nationale étant interdépendantes et indissociables, tout progrès européen est nécessairement le reflet de la qualité des évolutions nationales. Au regard des standards allemands en la matière, une planification plus centralisée à l'échelon européen ne se révèle nullement nécessaire.

Dans certains Etats membres, le financement du développement des réseaux entraîne une forte hausse des frais des transmissions et donc aussi du prix du kWh. Pour cette raison, le nouveau Règlement TEN-E du 17-04-2013 prévoit dans son article 12 un mécanisme de répartition transfrontalière des coûts d'investissement dans le cadre d'un projet d'intérêt commun : "les coûts d'investissement engagés efficacement... sont supportés par les GRT [i.e. gestionnaires de réseaux] concernés ou par les promoteurs d'infrastructures de transport des Etats membres sur lesquels l'incidence nette du projet est positive et, dans la mesure où ils ne sont pas couverts par les recettes provenant de la congestion ou d'autres frais, sont payés par les utilisateurs des réseaux moyennant les tarifs d'accès aux réseaux dans ces Etats membres ॥ (art. 12, § 1). A première vue, et sous l'angle des dommages et intérêts, cette disposition semble adaptée. Elle n'en risque pas moins de mettre en danger la planification conjointe des réseaux par le GRT. Car jusqu'ici prévalait le principe selon lequel c'est l'utilisateur du réseau situé dans son pays de résidence qui couvre les frais induits par la construction d'une ligne. Ce principe faisait consensus entre les GRT et était à la base même de leur programmation des réseaux comme de leurs accords de tarification.
Développer la

coopération franco-allemande...

... de même que celle des gestionnaires de réseaux dans I'UE

Nul besoin de planification centralisée à l'échelon de I'UE

Effets pervers de la nouvelle répartition transfrontalière des coûts d'investissement 
L'idée d'une telle répartition risque de donner lieu dans la réalité à une pratique de marchandage et ainsi de mettre en danger la bonne intelligence qui caractérisait la programmation des réseaux. Ce mécanisme n'apporte rien non plus en ce qui concerne l'accélération de la réalisation des projets, puisqu'il prévoit de lourdes procédures de coordination entre les GRT qui accroissent la complexité des procédures administratives pour les autorités de régulation. En effet, dans le cas où ces dernières ne parviendraient pas à un accord, c'est à l'Agence de coopération ACER qu'il reviendrait de décider de la répartition des coûts. Par ailleurs, dans la mesure où sont ainsi répartis entre les GRT de l'UE des coûts que supportent les utilisateurs du pays concerné, cette disposition touche des intérêts multiples, et on peut s'attendre à de nombreuses saisines des différentes juridictions nationales ou européennes (CJUE), et même de l'ACER. Au défi traditionnel qu'est l'adhésion de la population au développement du réseau dans chaque pays vient ainsi s'en ajouter un autre : quel utilisateur sera prêt à contribuer au financement, même partiel, d'un projet d'extension situé hors de son pays?

LA TRANSITION ÉNERGÉTIQUE COMME L'EXTENSION DES RÉSEAUX ÉLECTRIQUES SOnt des projets d'envergure européenne. Ils s'influencent mutuellement et s'interpénètrent, tant au niveau national que communautaire. Mieux les gestionnaires de réseaux coopèrent, plus ces projets seront couronnés de succès. Alors que jusqu'à présent, la programmation européenne de réseaux a fortement progressé, il serait regrettable que cette récente option pour la redistribution des coûts d'investissement au sein de l'UE génère des dissensions et ralentisse encore un processus de développement des réseaux déjà trop lent. Une adaptation des réseaux est nécessaire depuis toujours pour garantir la sécurité de l'approvisionnement ; les transitions énergétiques nationales et européenne se contentent finalement de changer les paramètres de la production et du transport le problème de fond de la sécurité de l'approvisionnement demeure.

Reste à mieux mettre en concordance la programmation des réseaux et la planification de la production dont l'impact sur les réseaux électriques a été démontré ici. La Bundesnetzagentur et son homologue français, la Commission de régulation de l'énergie (CRE) viennent de s'accorder en juillet 2014 sur une coopération portant sur les questions relatives aux marchés de capacités. Une telle intensification des échanges entre les régulateurs des deux pays, qui a pour objectif d'améliorer la sécurité de l'approvisionnement, est de nature à apporter une contribution non négligeable à la réalisation de la transition énergétique en Allemagne comme en France.

Traduction : I. Bourgeois

\section{Indications bibliographiques}

\footnotetext{
-Assemblée nationale, Projet de loi relatif à la transition énergétique pour la croissance verte (www.assemblee-nationale.fr/14/projets/pl2188.asp)

-BouRgeoIs I., «La transition énergétique allemande : mission impossible ? », Regards sur l'économie allemande, $n^{\circ} 112 / 2014$

-Bundesbedarfsplangesetz du 23 juillet 2013 (BGBI. I S. 2543; 2014 I S. 148), amendée par l'art. 11 de la loi du 21 juillet 2014 (BGBI. I S. 1066)

-BUndesministerium Für WIRTSCHAFt UND ENERgie, 10 Punkte Energie Agenda, 26-06-2014 (www. bmwi.de/BMWi/Redaktion/PDF/0-9/10-punkte-energie-agenda,property=pdf,bereich=bmwi2012,sprache=de,rwb=true.pdf) -BUNDESMINISTERIUM FÜR WIRTSCHAFT UND ENERGIE, EEG-Reform, 27-06-2014 (www.bmwi.de/DE/ Themen /Energie/Erneuerbare-Energien/eeg-reform,did=617412.html)

-BUNDESNETZAGENTUR, Bericht der Bundesnetzagentur an das Bundesministerium für Wirtschaft und Technologie, Auswirkungen des Kernkraftwerk-Moratoriums auf die Übertragungsnetze und die Versorgungssicherheit, 11-04-2011 (www.bmwi.de/BMWi/Redaktion/PDF/Publikationen/auswirkungen-kernkraft werk-moratorium-auf-uebertragungsnetze-und-versorgungssicherheit,property=pdf,bereich=bmwi2012,sprache=de,rwb=tr ue.pdf)

-BUNDESNETZAGentur, Bericht gemäß $§ 63$ Abs. 4a EnWG zur Auswertung der Netzzustands- und Netzausbauberichte der Elektrizitätsübertragungsnetzbetreiber, 08-01-2008 (www.bundesnetzagentur. de/cae/servlet/contentblob/31282/publicationFile/1104/BerichtNetzzustandNetzausbauld12385pdf.pdf)
} 
-Bundesnetzagentur, Monitoringbericht 2007 et Monitoringbericht 2009 (www.bundesnetzagentu r.de/cae/servlet/contentblob/31288/publicationFile/1118/Monitoringbericht2007ld12086pdf.pdf et www.bundesnetzagent ur.de/cae/servlet/contentblob/134810/publicationFile/1107/Monitoringbericht2009Energield17368pdf.pdf)

-BUNDESNetZAGENTUR, Genehmigung des Szenariorahmens für den Netzentwicklungsplan 2014 und den Offshore-Netzentwicklungsplan 2014, 30-08-2013 (www.netzausbau.de/cln_1412/DE/Bedarfsermit tlung/ Charlie/SzenariorahmenCharlie/SzenariorahmenCharlie-node.html)

-BUNDESNETZAGENTUR, « Bundesnetzagentur bestätigt den Reservekraftwerksbedarf für das Winterhalbjahr 2014/2015 und die Jahre 2015/2016 und 2017/2018 », communiqué de presse du 0205-2014 (www.bundesnetzagentur.de/cIn_1412/SharedDocs/Pressemitteilungen/DE/2014/140502Reservekraftwerksbe darfWinter2014-2018.html?nn=265778)

-BUNDESNETZAGENTUR, «Bundesnetzagentur und französische Regulierungsbehörde CRE plädieren für abgestimmte Energiemarktaufsicht », communiqué de presse du 04-07-2014 (www.bundes netzagentur.de/SharedDocs/Downloads/DE/Allgemeines/Presse/Pressemitteilungen/2014/140704_CRE.pdf?_blob=publi cationFile\&v=3) et, en français : www.cre.fr/documents/presse/communiques-de-presse/\%28annee\%29/2014

- COMMISSARIAT GÉNÉRAL À LA STRATÉGIE ET À LA PROSPECTIVE, La crise du système électrique européen. Diagnostic et solutions, Rapports \& Documents, janvier 2014 (www.strategie.gouv.fr)

- Commission EUROPÉENNE, Communication from the Commission to the European Parliament, the Council, the European Economic and Social Committee of the Regions, A policy framework for climate and energy in the period from 2020 to 2030 \{SWD(2014) 15 final\}\{SWD(2014)16 final\}, Bruxelles, 22-01-2014 COM(2014) 15 final (http://ec.europa.eu/energy/doc/2030/com_2014_15_en.pdf)

- Commission eURopéenNe, Communication from the Commission to the European Parliament and the Council, Energy Efficiency and its contribution to energy security and the 2030 Framework for climate and energy policy, Bruxelles, 23-07-2014 COM (2014)520 final (http://ec.europa.eu/energy/effi ciency/events/doc/2014_eec_communication_adopted.pdf)

- Commission EURoPÉENNE, Communication from the Commission to the European Parliament and the Council, European Energy Security Strategy, Bruxelles, 28-05-2014, COM (2014) 330 final (http://ec.europa.eu/energy/doc/20140528_energy_security_communication.pdf)

- Commission eURopÉenne, Communication de la Commission au Parlement, au Conseil, au Comité économique et social européen et au Comité des Régions, Feuille de route vers une économie compétitive à faible intensité de carbone à l'horizon 2050, \{SEK(2011) 287 final\} \{SEK(2011) 288 final\} \{SEK(2011) 289 final\}, Bruxelles, 08-03-2011 KOM(2011) 112 final (http:/leurlex.europa.eu/ LexUriServ/LexUriServ.do?uri=COM:2011:0112:FIN:fr:PDF)

-Commission eUROPÉENNE, Règlement délégué (UE) n 1391/2013 de la Commission du 14 octobre 2013 modifiant le règlement (UE) $n^{\circ}$ 347/2013 du Parlement européen et du Conseil concernant des orientations pour les infrastructures énergétiques transeuropéennes, en ce qui concerne la liste des projets d'intérêt commun de I'Union (http://eur-lex.europa.eu/legal-content/FR/TXT/PDF/?uri=CELEX: 32013R 1391\&from=EN)

-Conseil de L'Union eURopÉenne, Marché intérieur de l'énergie, Rapport du Conseil, ENER 584, 12-12-2013, 17755/13 (http://register.consilium.europa.eu/doc/srv?!=FR\&f=ST\%2017755\%202013\%20INIT)

-CONSEIL EUROPÉEN, Barcelone, 15/16-03-2002, Conclusions (http://www.consilium.europa.eu/ueDocs/cms _Data/docs/pressdata/de/ec/71067.pdf)

-Décision n 1364/2006/CE du Parlement européen et du Conseil du 6 septembre 2006 établissant des orientations relatives aux réseaux transeuropéens d'énergie et abrogeant la décision 96/391/CE et la décision $n^{\circ}$ 1229/2003/CE (http://eur-lex.europa.eu/legal-content/FR/TXT/PDF/?uri=CELEX: 32006D1364\& from $=\mathrm{FR}$ )

-Energieleitungsausbaugesetz du 21 août 2009 (BGBI. I S. 2870)

-Energiewirtschaftsgesetz du 7 juillet 2005 (BGBI. I S. 1970, 3621), amendée par l'art. 6 de la loi du 21 juillet 2014 (BGBI. I S. 1066)

-ENTSO-E, Ten-Year Network Development Plan 2014, 10-07-2014 (www.entsoe.eu/major-project s/tenyear-network-development-plan/tyndp-2014/Pages/default.aspx)

-FIScher S., «Les défis de l'intégration européenne pour la politique énergétique allemande » $R e-$ gards sur l'économie allemande, $n^{\circ}$ 90/2009

-IEA, World Energy Investment Outlook. Special Report, Londres, 03-06-2014

-Netzausbaubeschleunigungsgesetz Übertragungsnetz du 28 juillet 2011 (BGBI. I S. 1690), amendée par l'art. 4 de la loi du 20 décembre 2012 (BGBI. I S. 2730)

-NiES S., At the speed of light, Electricity Interconnections for Europe, IFRI, Paris, Bruxelles, 2009 (www.ifri.org/downloads/interconnectionbis_1.pdf)

-Règlement (UE) n ${ }^{\circ}$ 347/2013 du Parlement européen et du Conseil du 17 avril 2013 concernant des orientations pour les infrastructures énergétiques transeuropéennes, et abrogeant la décision $n^{\circ} 1364 / 2006 / C E$ et modifiant les règlements (CE) $n^{\circ} 713 / 2009$, (CE) $n^{\circ} 714 / 2009$ et (CE) $n^{\circ} 715 /$ 2009 (http://eur-lex.europa.eu/legal-content/FR/TXT/PDF/?uri=CELEX:32013R0347\&from=FR)

-SÉNAT, Rapport d'information de M. Jean BIZET, fait au nom de la commission des affaires européennes $n^{\circ} 534$ (2013-2014) le 14 mai 2014 (www.senat.fr/rap/r13-534/r13-534.html)

-UCTE, Transmission Development plan Development of interconnections, avril 2009, Bruxelles (www.entsoe.eu/ fileadmin/user_upload/_library/publications/ce/otherreports/tdp09_report_ucte.pdf)

-www.netzausbau.de/cln_1422/DE/Mitreden/Veranstaltungen/Veranstaltungen-node.html. 